\title{
BEAM PARAMETERS OF THE AGS SYNCHROTRON DURING FAST BEAM EXTRACTION AT THE LOCATION OF THE AGS KICKER*
}

\author{
N.Tsoupas, L.Ahrens, W. Glenn, W.Van Asselt, M. Brennan, M. (Sanki) Tanaka, K. Smith, and K. Brown \\ BNL Upton NY 11934 USA
}

\begin{abstract}
The longitudinal requirements for the AGS to RHIC transfer process requires a precise control of the radius and frequency of the circulating bunches in the AGS just before the bunch extraction from the AGS and subsequent injection into RHIC via the ATR transfer line [1]. In addition the transverse beam parameters in the AGS at the location of the Extraction kicker define the "beammatching" between the ATR line and RHIC. In this paper we present theoretical and experimental results related to the longitudinal and transverse parameters of the AGS.
\end{abstract}

\section{INTRODUCTION}

The Alternating Gradient Synchrotron (AGS) of the Brookhaven National Laboratory (BNL) is the injector to the Relativistic Heavy Ion Collider (RHIC). Currently the AGS synchrotron extracts beam bunches of $\sim 10^{9} \mathrm{Au}$ ions/bunch at a beam energy corresponding to a $\gamma$-value of $\sim 10.5$ to fill 56 buckets (out of 60) of the "Blue Ring" and 56 buckets of the "Yellow Ring" of RHIC. The injected beam bunches in both "Blue Ring" and "Yellow Ring" are subsequently accelerated to an energy corresponding to a $\gamma$-value of $\sim 105$ and are brought into collision at any of the six interaction points of the RHIC collider. A primary task during beam injection into RHIC is the "beam-matching" of the transverse beam parameters at the injection point of RHIC and the synchronization of the injected beam-bunch from the AGS to the buckets in RHIC. This paper is concerned with: a) the value of the beam parameters and their functional dependence on the beam momentum (average radius of circulating beam in AGS) at a particular azimuthal location (the location of the G10-extraction-kicker of AGS ), at the time of beam extraction b) the connection of these beam parameters on the beam parameters at the origin of the ATR transfer line and c) the effect of the extraction "local-beam-bumps" in AGS on the synchronization of the extracted bunches from AGS to the buckets of RHIC.

\section{SCOPE OF THE STUDY}

Theoretical calculations of the beam parameters of AGS during beam extraction from the AGS, predict a tune $\left(Q_{x, y}\right)$ shift of the AGS and an increase of the beam path length of the circulating beam $(\sim 1.5 \mathrm{~cm})$ when the extraction-bumps of the AGS are turned on. They also predict a rather strong dependence of the dispersion functions $\eta_{\mathrm{x}}$, and $\eta_{\mathrm{x}}$, on the average radius (momentum) of the circulating beam bunches in the AGS, at the location of the AGS fast extraction kicker. This behavior of the dispersion function which is attributed to the field of the chromaticity sextupoles, and the sextupole of the AGS main magnets, that the beam samples when the extraction beam bumps are turned on, prompted an experimental study in the AGS in order to gain knowledge on the momentum dependence of the physical quantities of tune $\mathrm{Q}_{\mathrm{x}, \mathrm{y}}$, and dispersion function $\eta_{\mathrm{x}}$ which are relevant in the beam matching between the AGS-to-RHIC (ATR) transfer line and RHIC. In this paper we present data from the theoretical calculations and compare them with those derived from the measurements. In addition, when the beam-bumps are turned on, they interact with other main magnets of the ring and alter the current of the Main Magnet Power Supply. Details of interactions with the power supplies and the RF system will be presented and a solution will be given.

\section{BEAM PARAMETERS IN AGS}

In this section we discuss the following items a) the mechanism of the Fast Beam Extraction (FEB) as applied to the AGS, b) the method to calculate the beam parameters at azimuthal locations of the AGS, c) the results obtained from the calculations in part (b) above.

\subsection{Mechanism of the FEB}

While the beam bunches circulate in the AGS at a $\mathrm{p} / \mathrm{q}$ $\sim 26 \mathrm{GeV} / \mathrm{c}$ and just before bunch extraction from the AGS, the beam is "locally bumped" at two sections of the AGS ring $[2,3,4]$. One local bump ("G09") includes the location of the G10-kicker[2], and the other local-bump ("H11") at includes the location of the H10-septum[2] where the beam circulates $\sim 10 \mathrm{~mm}$ away from the septum.

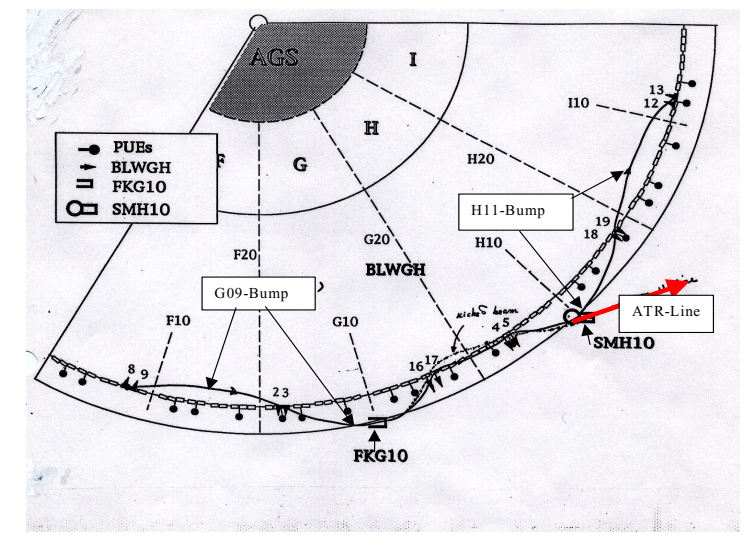

Figure 1: Schematic diagram of the AGS's FEB section. 
Figure 1 is a schematic diagram of the FEB section of the AGS showing the location of the G10-kicker (FKG10) the location of the H10-septum (SMH10), the location of the magnets which generate the "local-bumps", the orbit of the circulating "bumped-beam", (note the "G09" and "H11" bumps), and the trajectory of the extracted beam into the ATR transfer line.

When the beam is at its maximum "bumped-amplitude" the G10-kicker fires and the beam is kicked inside the high field region of the H-10 septum magnet and subsequently is extracted to the origin of the ATR line.

\subsection{Calculation of Beam Parameters}

The code AGS_BATE (AGS Beam Acceleration Transport and Extraction) [3] was used to calculate the beam parameters at any azimuthal location in AGS and at the beginning of the ATR line. Details of the procedure are discussed in refs. $[3,4]$.

\subsection{Presentation of theoretical results}

Figure 3 is a plot of the values of the functions $\beta_{x, y}$ at the middle of the straight section SS-130 which is the location of G10 kicker.

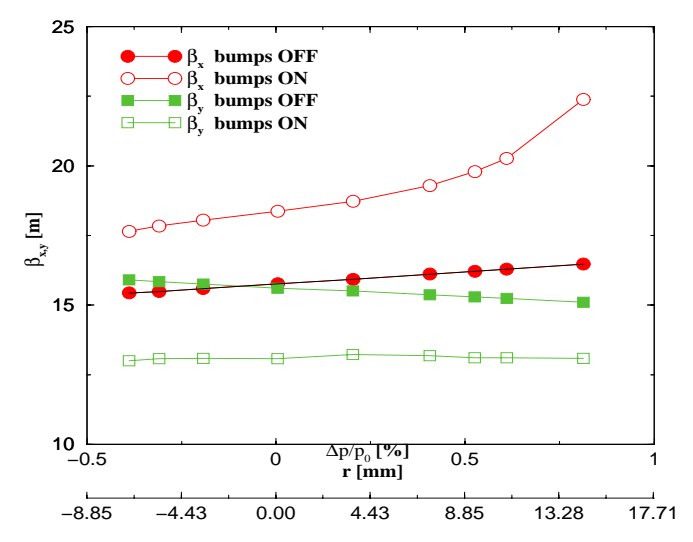

Figure 3: The functions $\beta_{\mathrm{x}, \mathrm{y}}$ at the location of the kicker.

The $\beta_{x, y}$ is plotted as a function of the quantity $\Delta \mathrm{p} / \mathrm{p}_{0}=\left[\mathrm{p}(\mathrm{r})-\mathrm{p}\left(\mathrm{r}_{0}\right)\right] / \mathrm{p}\left(\mathrm{r}_{0}\right)$ where $\mathrm{p}\left(\mathrm{r}_{0}\right)$ is the momentum of a particle describing the ideal closed orbit with average radius $\mathrm{r}_{0}$ and $\mathrm{p}(\mathrm{r})$ is the momentum of a particle describing a closed orbit with average radius ${ }^{1} \mathrm{r}$. The function $\beta_{\mathrm{x}, \mathrm{y}}$ is also plotted as function of the average radius (note the lower $\mathrm{x}$-scale of the plot). There is a strong dependence of $\beta_{\mathrm{x}}$ function on the beam momentum when the beam is locally bumped. This dependence is attributed to the sextupole field of the chromaticity sextupoles and the sextupole introduced by the main magnets located at the region of the local-beam-bump. This sextupole field becomes significant when the beam is displaced from the ideal orbit, as is the case for the bumped-beam. This sextupole field can also account for

\footnotetext{
${ }^{1}$ The average radii are in reference to the closed orbits with bumps OFF
}

the change of the horizontal and vertical chromaticities when the bumps are turned on. The chromaticities are calculated from the slope $\left\{\xi_{\mathrm{x}, \mathrm{y}}=\left(\Delta \mathrm{Q}_{\mathrm{x}, \mathrm{y}}\right) /\left(\mathrm{Q}_{\mathrm{x}, \mathrm{y}} \Delta \mathrm{p} / \mathrm{p}_{0}\right)\right\}$ of the tunes $\mathrm{Q}_{\mathrm{x}, \mathrm{y}}$ which are plotted in figure $4 \mathrm{vs} \Delta \mathrm{p} / \mathrm{p}_{0}$.

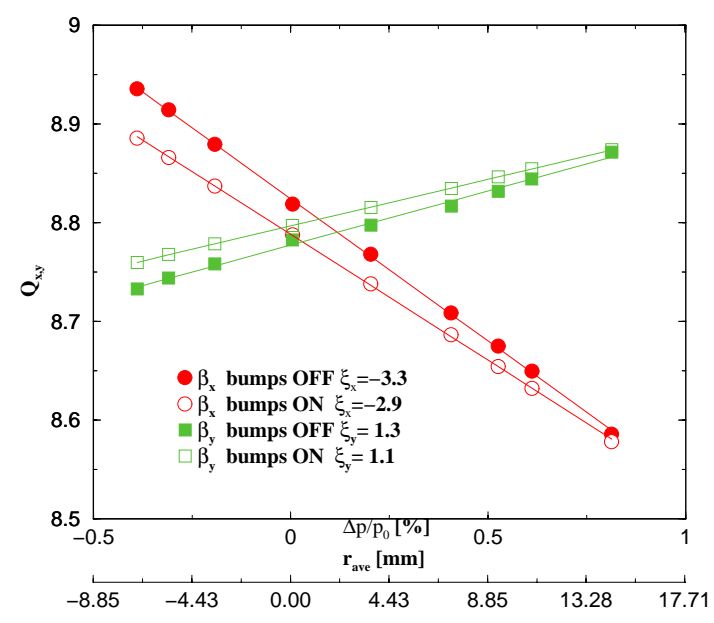

Figure 4: The Calculated Hor. and Vert. Tunes

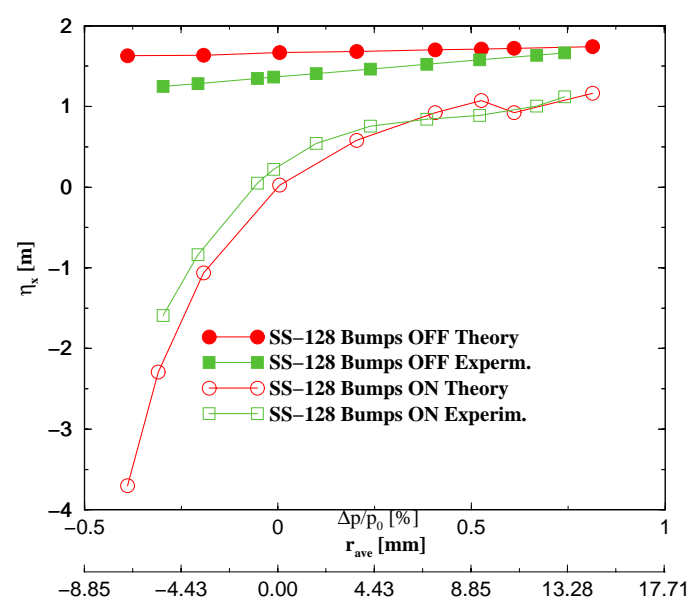

Figure 5: The function $\eta_{\mathrm{x}}$ at straight section ${ }^{2}$ SS-128 with Bumps ON and OFF as a function of momentum. The theoretical data are shown in circles and the experimental data in squares. The function $\eta_{\mathrm{x}}$ exhibits a dependence on the beam momentum when the local beam bumps are on.

\subsection{Measurements of Beam Parameters}

The measured physical quantities are a) the dispersion function at the straight sections SS-128, and b) the horizontal and vertical tunes of the AGS, both as a function of the beam's momentum $\left(\mathrm{r}_{\mathrm{ave}}\right)$.

The measured dispersion which is plotted in figure 5 as a function of beam momentum exhibits similar trend as the the calculated dispersion shown in the same figure.

The measured horizontal and vertical tunes of the AGS with the bumps on and off are plotted in figure 6 as a function of beam momentum. The change of the AGS

\footnotetext{
2 This is the closest azimuthal location to the G10 kicker where dispersion can be measured experimentally.
} 
chromaticity when the bumps are turned on indicates the presence of sextupole field.

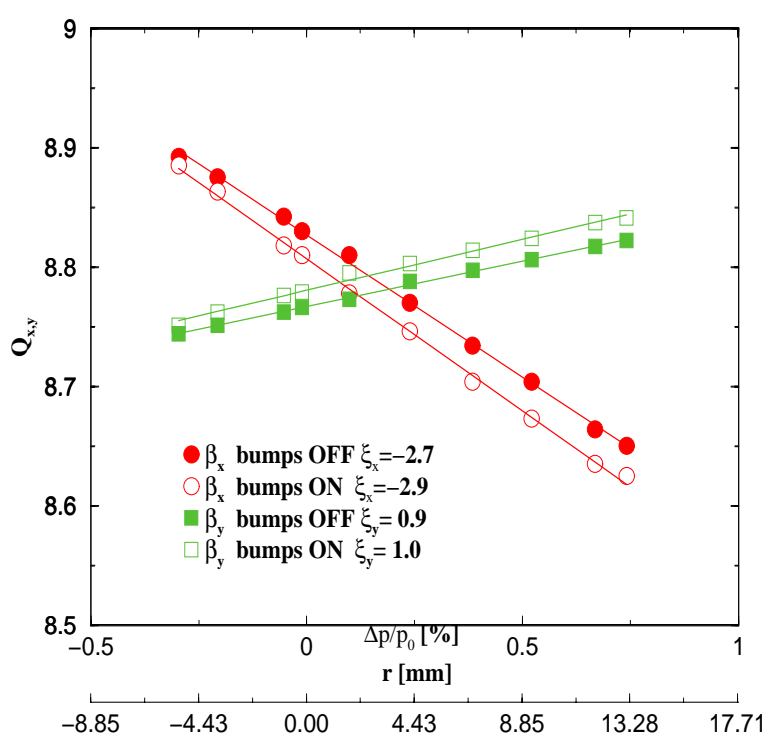

Figure 6: The measured hor. and vert. tunes of AGS

\subsection{Beam Parameters at the Origin of ATR Line}

The beam parameters at the origin of ATR as a function of the $r_{\text {ave }}$ have been calculated using the theoretical data of this paper. The $\beta_{x, y}$ and $\alpha_{x, y}$ functions exhibit small dependence on $r_{\text {ave }}$ (see Table I).

Table I: Extreme values for the $\beta_{\mathrm{x}, \mathrm{y}}$ and $\alpha_{\mathrm{x}, \mathrm{y}}$ functions.

\begin{tabular}{|c|c|c|c|c|}
\hline & $\alpha_{x}$ & $\beta_{\mathrm{x}}[\mathrm{m}]$ & $\alpha_{y}$ & $\beta_{\mathrm{y}}[\mathrm{m}]$ \\
\hline $\mathrm{r}_{\mathrm{ave}} \min \quad-6 \mathrm{~mm}$ & -3.12 & 29.2 & 1.14 & 8.45 \\
\hline $\mathrm{r}_{\mathrm{ave}} \max 14 \mathrm{~mm}$ & -3.44 & 32.7 & 1.16 & 7.90 \\
\hline
\end{tabular}

There is however a strong dependence of the $\eta_{x}$ and $\eta^{\prime}{ }_{x}$ functions on $r_{\text {ave }}$ as shown in figure 7 which plots these functions as a function of $r_{\text {ave }}$. The arrow in figure 7 indicates the value of the $r_{\text {ave }}$ during RHIC operations.

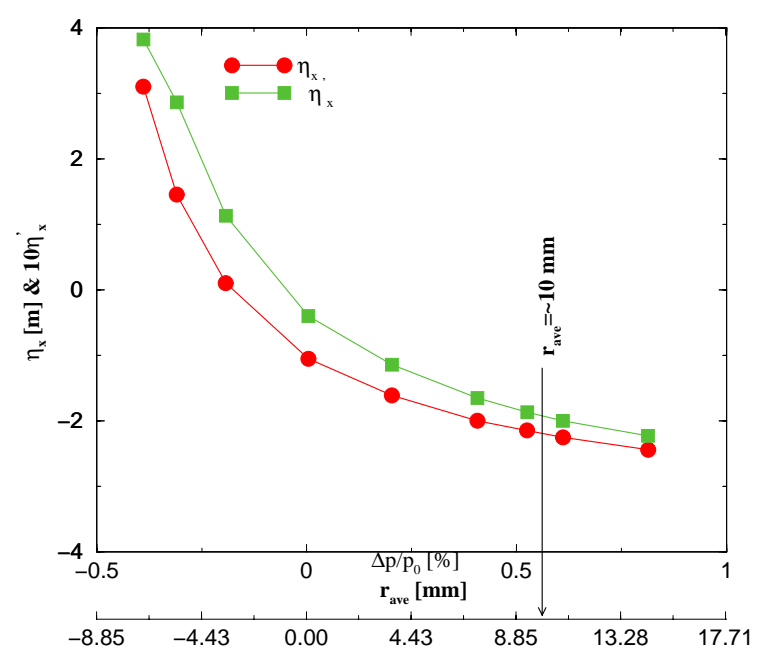

Figure 7: Calculated $\eta_{x} \& \eta_{x}^{\prime}$ functions at the origin of the ATR line as function of the $r_{\text {ave }}$ of AGS.

\section{EFFECT OFF THE BUMPS ON THE RF}

In order to synchronize the extracted bunches from the AGS to the RHIC's circulating buckets, the AGS RF frequency and the $r_{\text {ave }}$ of the circulating beam during the extraction must have specified values. When the "local bumps" $[2,3,4]$, are powered, a) Increase of the path length of the beam's orbit by $\sim 1.5 \mathrm{~cm}$ (this increases the RF freq.), and $b$ ) increase the main magnet field by $\sim 10$ Gauss (this decreases the $r_{\text {ave }}$ ). These effects tend to cancel each other but not completely, with the increase in frequency dominating the total effect.

Since the field of the main magnet does not rise enough, the RF decelerates the beam slightly to keep the phase constant. Figure 8 summarizes the behavior of some parameters during extraction. The top trace in figure 8 is the waveform of the current which excites the "localbumps". The second trace from the top plots the increase of the current of the main AGS magnet. The third trace plots the variation of $r_{\text {ave }}$ which is required to keep the phase between the RF frequency and the buckets constant. Pulsed DC power supplies are on order to hold the beam steady during extraction.

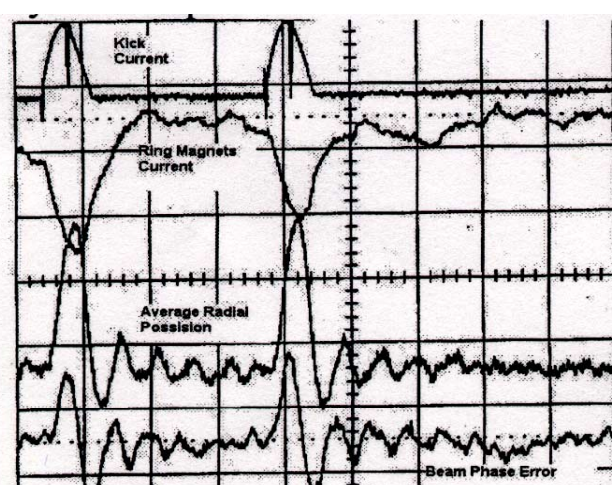

Figure $\mathrm{x}$ : variation of physical quantities in extraction

\section{CONCLUSIONS}

The "local-beam-bumps" used in the AGS for the fast beam extraction affect the beam parameters in AGS and the dispersion functions $\eta_{x}$ and $\eta^{\prime}{ }_{x}$ at the origin of the ATR line. Alternative set-up of the AGS during beam extraction. that reduces the sextupole effect may remove the dependence of $\eta_{x}$ and $\eta_{x}^{\prime}$ on the radius of the AGS.

\section{REFERENCES}

[1] N. Tsoupas et. al. BNL, "Focusing and Matching Properties of the ATR Transfer Line" PAC97 Vancouver, BC, May 12-16 (1997)

[2] M. Tanaka "The New Fast Extraction System at the AGS" AGS/AD/Tech. Note No. 347

[3] N. Tsoupas et. al. "Fast Extracted Beam (FEB) for the g-2 Experiment" C-A/AP/54

[4] N. Tsoupas "Closed Orbit Calculations at AGS and

Extraction Beam Parameters at H13" AD/RHIC/RD-75

[5] G. H. Morgan, "Fortran IV Version of Beam; The AGS Orbit Computing Program”, AGS Int.Rep. 